\title{
PANCREATITE NECRO-HEMORRÁGICA: ATUALIZAÇÃO E MOMENTO DE OPERAR
}

\author{
Necrotizing pancreatitis: update and when to operate \\ Ricardo Antonio REFINETTI, Rodrigo MARTINEZ
}

Trabalho realizado no Departamento de Cirurgia da Faculdade de Medicina da Universidade Federal do Rio de Janeiro, UFRJ, RJ, Brasil

DESCRITORES - Pancreatite. Procedimentos cirúrgicos operatórios. Necrose.

\section{Correspondência: \\ Ricardo Antonio Refinetti, ricardoar@hucff.ufrj.br}

Fonte de financiamento: não há Conflito de interesses: não há

Recebido para publicação: 06/10/2009 Aceito para publicação: 15/01/2010

HEADINGS - Pancreatitis. Surgical procedures, operative. Necrosis.
RESUMO - Introdução - A pancreatite necro-hemorrágica representa a variante mais grave do espectro de apresentações clínicas que podem compor o quadro da pancreatite aguda. Embora já conhecida há muitos séculos, inúmeras questões permanecem em aberto acerca dessa entidade e o número de trabalhos sobre o assunto publicados nos últimos anos tem sido muito expressivo. Método: Foi realizada ampla pesquisa na literatura, com especial atenção aos artigos publicados nos últimos três anos e indexados ao PubMed. Foram utilizados os seguintes descritores de forma cruzada: pancreatitis, surgical procedures; necrosis. A pesquisa inicial evidenciou cerca de 13.000 artigos, sendo avaliados os mais relevantes dos últimos três anos além de artigos mais antigos, considerados "clássicos" sobre o assunto e que, portanto, não poderiam deixar de ser citados. Conclusão - O tratamento da pancreatite aguda envolve um grande número de questões, dentre as quais as mais importantes estão relacionadas ao manejo da antibioticoterapia, tipo de dieta empregada e as questões relacionadas ao manejo da necrose infectada. Em especial, mudanças radicais foram implementadas nos últimos anos sobre todos esses tópicos, e uma atualização constante deve ser obrigatoriamente buscada pelos profissionais envolvidos no tratamento dessa doença.

ABSTRACT - Background - Necrotizing pancreatitis represents the most severe form of presentation from the clinical spectra of acute pancreatitis. Although known for many centuries, many questions remain open about this entity and a great number of articles were published about this matter in the last few years. Method - A throughout research in the literature, with special attention to the articles published in the last three years and indexed to the PubMed was performed. The following headings were used: pancreatitis, surgical procedures, necrosis. The initial research rendered about 13000 articles, and the ones published in the last three years were evaluated. Some older, but remarkable articles were also included given their importance to this matter. Conclusion - The treatment of acute pancreatitis involves a great number of questions, among which the most important are the ones related to the use of antibiotics, type of diet employed and the questions related to the manangement of the infected necrosis. There were many radical changes instituted in the last years on all these topics and a constant updating must be necessarily done by the ones involved on the treatment of this disease.

\section{INTRODUÇÃO}

A Pancreatite Aguda (PA) é doença com expressão clínica muito $\triangle$ variável, podendo manifestar-se desde um simples desconforto abdominal, sem grandes repercussões sistêmicas ao paciente, até um quadro muito mais florido, marcado majoritariamente pela falência múltipla de órgãos e morte ${ }^{20}$.

Os avanços observados nos últimos anos em relação a ela e suas complicações - com grande número de publicações clínicas e experimentais -, permitiu melhor conhecimento de sua evolução, fazendo com que se obtivessem melhores resultados no seu tratamento. Mas, apesar de todos os avanços experimentados ao longo destes anos, a fisiopatologia da PA e de suas complicações permanece com muitos pontos obscuros, o que em muito 
prejudica a elaboração de propostas terapêuticas de sucesso para essa doença ${ }^{20}$.

Ela é doença inflamatória do pâncreas, associada à diversas condições, conhecidas como agentes etiológicos da pancreatite aguda, sendo que a doença litiásica biliar e a ingestão de álcool concorrem com cerca de $80 \%$ dos casos, variando conforme a região que se estuda ${ }^{1}$.

Além destes principais agentes etiológicos, pode-se citar certas drogas como a azatioprina, sulfonamidas, pentamidina e ácido valpróico, com características idiossincráticas, as tiazidas, quando em altas doses e o estrogênio que leva a grande aumento nos níveis séricos de trigicerídeos. As infecções, as hiperlipidemias, as hipercalcemias, o trauma e a hipotensão, principalmente em operações cardíacas e no pós-parto, também podem levar à pancreatite aguda. Deve-se lembrar ainda que em cerca de $10 \%$ dos casos não se consegue determinar a causa que levou à pancreatite ${ }^{20}$.

\section{MÉTODO}

Foi realizada ampla pesquisa na literatura, com especial atenção aos artigos publicados nos últimos três anos e indexados ao PubMed. Foram utilizados os seguintes descritores de forma cruzada: pancreatitis, surgical procedures; necrosis. A pesquisa inicial evidenciou cerca de 13.000 artigos, sendo avaliados os mais relevantes dos últimos três anos, em que os textos completos estivessem disponíveis para leitura, além de artigos mais antigos, considerados "clássicos" sobre o assunto e que, portanto, não poderiam deixar de serem citados.

\section{Terminologia relevante}

Dentro das inúmeras formas de apresentação da $\mathrm{PA}$, seguramente aquela que representa o maior desafio terapêutico é a pancreatite aguda necrohemorrágica. Embora essa entidade tenha já sido descrita primeiramente em 1652, sua devastadora apresentação clínica e os frustrantes resultados terapêuticos apresentados até o momento fazem dela ainda uma das doenças mais estudadas nos dias atuais ${ }^{7}$. Nessa conceituação inicial, já encontra-se um grande problema, uma vez que a terminologia adotada pelos diferentes autores é bastante variável, o que dificulta até mesmo a comparação dos resultados obtidos entre os diferentes grupos e a aceitação de condutas uniformes. Todavia, de uma forma geral, esses quadros correspondem cerca de 15 a 20\% dos casos de PA, e sua incidência vem crescendo não apenas por acompanhar o crescimento na sua incidência de uma forma geral, mas também pela constatação de que a evolução no suporte intensivo e manejo dos pacientes graves permitiu maior sobrevida dos pacientes portadores dessa variante ${ }^{9}$.

Em linhas gerais, o quadro clínico da PA grave pode ser dividido em dois estágios principais. Em um primeiro momento - que geralmente compreende a primeira semana de doença -, verifica-se quadro de extensa síndrome de resposta inflamatória sistêmica, e nessas condições a morbi-mortalidade está mais associada às complicações decorrentes dessa condição clínica geral. Da mesma forma, nessa etapa, os desafios terapêuticos se entrelaçam com os de inúmeras outras doenças, caracterizadas por uma cascata de eventos iniciais comuns, como a sepse grave. Mais tardiamente, a partir da segunda ou terceira semanas, prevalece a disfunção multiorgânica como consequência da infecção da necrose pancreática ${ }^{4}$.

Desta forma, várias classificações foram historicamente utilizadas, dando origem a uma terminologia muito ampla e que não raro gera confusões (Sarles 1965 - Marselha I, Singer 1984 Marselha II,1985 - Cambridge). Com o objetivo de se por fim a esse problema, propôs-se Classificação de Atlanta - 1992, que tem como principais elementos ${ }^{8,45}$ :

1 - Pancreatite Leve, caracterizada por quadro pouco florido, e em cujos pacientes não há necessidade da manipulação do parênquima pancreático.

2 - Pancreatite Grave, definida como todo caso de pancreatite associado à falência orgânica e/ou a complicações locais, a saber: a) coleções líquidas definidas como aquelas que se formam logo após o início da pancreatite, não tem cápsula própria; b) pseudocistos - resultantes da evolução das coleções fluidas agudas, já que após algumas semanas há tendência para formação de uma pseudo cápsula cujas paredes são os próprios órgãos adjacentes; c) necrose - resultante da auto-digestão do parênquima pancreático e da gordura adjacente.

Tanto o pseudocisto, quanto a necrose podem ser focos de infecção. O pseudocisto infectado não existe como terminologia própria na classificação de Atlanta, sendo assim classificado como um abcesso. Na mesma linha, a necrose que sofre liquefação, forma coleção e se infecta sendo também denominada de abcesso. O mecanismo principal de infecção é decorrente do aumento na permeabilidade intestinal, com consequente translocação de bactérias e proliferação no tecido necrótico. Nesse sentido, os principais agentes infecciosos são justamente os bacilos gram-negativos entéricos como a Escherichia coli e Klebisiella spp. bem como Enterococcus spp ${ }^{44}$. O emprego de antibióticos de amplo espectro nessas condições - tema que será melhor dissecado adiante -, vem provocando aumento nos casos de infecção por germes mais resistentes e até mesmo por agentes fúngicos 21,28 .

\section{Diagnóstico da pancreatite aguda grave}

A primeira etapa no sentido de se buscar o melhor tratamento da pancreatite aguda grave é a identificação daqueles que apresentam essa condição. Para isso, torna-se imperativo o uso de classificações específicas ou não da pancreatite aguda, como a classificação 
de Ranson, Glasgow e APACHE II (Acute Physiology and Cronic Health Care Evaluation II). Sendo assim, pacientes que apresentam escores elevados devem ser observados mais de perto, merecendo inclusive a internação em unidades de terapia intensiva ${ }^{10}$. Ainda com relação aos escores, a presença de três ou mais critérios de Ranson ou de uma pontuação de 8 ou mais na classificação de APACHE II são critérios para a definição de pancreatite aguda grave ${ }^{8}$. Da mesma forma, o emprego dos métodos de imagem é de valia para a classificação da gravidade da pancreatie (critérios de Balthazar). Em especial, o achado de áreas não-perfundidas na tomografia computadorizada com contraste venoso realizada após pelo menos $24-48 \mathrm{~h}$ após o evento agudo, ou seja, de necrose pancreática, é um dos de maior valia, uma vez que como já foi dito, essa é uma lesão com potencial grande de sofrer infecção secundária, além de sua extensão ser proporcional ao grau de disfunção orgânica ${ }^{27}$.

\section{Antibioticoterapia na PA grave}

Durante a avaliação do paciente com PA grave, o achado de necrose é seguramente um dos mais alarmantes. Além da já descrita correlação desta com a presença de disfunção de múltiplos órgãos, a necrose pancreática pode tornar-se infectada em uma porcentagem considerável dos casos, sendo mais comum nas necroses extensas, que acometem mais de $50 \%$ do órgão, quando então a taxa de infecção ultrapassa os $40 \%{ }^{6}$. Além disso, o diagnóstico da necrose infectada é também um grande desafio, já que pacientes portadores de necrose têm tipicamente quadro clínico grave, e podem apresentar-se sépticos, com febre e leucocitose ainda que não apresentem infecção. Além do mais, esses pacientes muitas vezes, por seu quadro debilitado, apresentam inúmeras fontes possíveis de infecção. Esses dados moveram a comunidade médica envolvida no tratamento dessa doença a recomendar o tratamento profilático com antibióticos de amplo espectro em pacientes com necroses pancreáticas de volumes significativos ${ }^{45}$. Ainda mais recentemente, essa questão tem sido contemplada por diversos trabalhos de desenhos experimentais heterogêneos. Em especial, algumas meta-análises recentes apresentaram resultados taxativos, não demonstrando benefício do emprego de antibioticoterapia nesses pacientes e, portanto, modificando os protocolos de tratamento ${ }^{14,26,32}$. De uma forma alternativa, tem-se proposto o uso de antibióticos não-absorvíveis por via oral (descontaminação digestiva seletiva) como uma forma de se reduzir as taxas de infecção de necrose pancreática, com resultados iniciais promissores ${ }^{30,41}$. Sendo assim, embora ainda seja tema de amplo debate ${ }^{20}$, a conduta mais aceita para a abordagem da necrose pancreática seria a do emprego de antibióticos quando do diagnóstico de certeza da presença de infecção na mesma. Esse diagnóstico pode ser feito, em algumas situações, a partir de métodos de imagem, como uma tomografia computadorizada de abdome evidenciando gás no interior da necrose ${ }^{1}$. Alternativamente, alguns marcadores bioquímicos como a proteína C reativa, a procalcitonina e a mieloperoxidase vem sendo testados como preditores de infecção, com índices de confiabilidade variáveis entre os estudos ${ }^{11,34}$. Da mesma forma, a deterioração da condição clínica/hematológica, a presença de hemoculturas positivas, em especial para agentes entéricos e testes sorológicos positivos para endotoxinas também são sugestivos de infecção. Ainda mais recentemente, um trabalho prospectivo grande evidenciou que parâmetros clínico-laboratoriais como leucocitose e proteína $C$ reativa elevada seriam eficazes em discriminar a necrose estéril da infectada ${ }^{13}$. Na maior parte das vezes, todavia, é necessária a realização de punção com cultura do material obtido, considerada o padrão-ouro para a confirmação diagnóstica, com acurácia de 89 a $100 \%{ }^{5}$ e cujo resultado por sua vez norteará a antibioticoterapia empregada ${ }^{10}$. A despeito disso, a punção da necrose estéril traz consigo o risco inerente de contaminação iatrogênica da mesma ${ }^{38}$, e sendo assim muitos centros optam pela realização desse procedimento apenas em condições de dúvida quanto ao diagnóstico dessa infecção.

\section{Manejo dietético}

O manejo da dieta nos pacientes com PA grave é também um tópico que sofreu grandes modificações ao longo das últimas décadas. Em especial, o conceito da importância do retorno da dieta enteral, como mecanismo antagonizador da translocação bacteriana e desta forma resultando em menor incidência de necrose infectada tem sido confirmado por diversos trabalhos heterogêneos, que demonstram também internações menos prolongadas e menos intervenções cirúrgicas em pacientes mantidos com dieta enteral através de sonda nasoenteral posicionada no jejuno ${ }^{31,33}$. Esses resultados contrapõem o conceito primitivo de que a alimentação enteral estimularia as secreções pancreáticas e desta forma poderia agravar os quadros de PA grave. Algumas questões permanecem em aberto, como o tipo de dieta enteral que seria melhor empregada nesses pacientes e a possibilidade de administração da mesma pela via gástrica. Além do mais, o momento de início dessa dieta parece ser um ponto que deve ser individualizado para cada paciente.

\section{Emprego de métodos endoscópicos}

$\mathrm{Na}$ PA biliar, uma questão bastante relevante a ser abordada diz respeito ao diagnóstico e tratamento da coledocolitíase, uma vez que a presença da mesma pode ser elemento fundamental não apenas causador, mas também perpetuador do processo inflamatório sobre o pâncreas. Por outro lado, sabe-se que em grande parte dos casos de pancreatite aguda biliar o cálculo causador do processo inflamatório já não mais está presente na via biliar ${ }^{47}$, e ao mesmo tempo, exames invasivos como a colangiopancreatografia 
endoscópica retrógrada (CPER) trazem consigo o risco de complicações devastadoras para os pacientes acometidos de PA grave. Desta forma, trabalhos já foram realizados propondo o emprego seletivo desse método em pacientes com PA biliar grave em momentos precoces da evolução da doença, com resultados conflitantes a respeito do real benefício da mesma nessas condições $3,16,18,36$. Além do mais, embora a ultrassonografia abdominal tenha sensibilidade demasiadamente baixa para o diagnóstico dessa condição, outros métodos mais recentes demonstraram maior eficácia para esse fim. Sendo assim, o diagnóstico da coledocolitíase é na atualidade preferencialmente realizado através de métodos pouco ou não-invasivos, como a ecoendoscopia e a colangioressonância nuclear magnética, cuja acurácia em alguns trabalhos alcança os níveis da CPER. Esse último exame, por sua vez está mais indicado quando o diagnóstico da coledocolitíase está confirmado ou tem alta probabilidade, como na presença de colangite, ou em paciente com níveis consideravelmente elevados de bilirrubinemia.

É também fundamental se citar o crescente papel dos procedimentos endoscópicos na drenagem e, mais recentemente, no debridamento de tecidos pancreáticos necrosados (operações por orifícios naturais - NOTES), como será melhor descrito mais adiante $^{19}$.

\section{O papel da cirurgia na PA grave}

Classicamente, a PA é condição de tratamento majoritariamente clínico ${ }^{1,2}$. A despeito disso, o cirurgião faz habitualmente parte da equipe multidisciplinar que acompanha esses casos, e há algumas situações nas quais a intervenção desse profissional está claramente indicada. Em especial, nas situações de dúvida diagnóstica, nas quais o paciente apresenta sinais clássicos de abdome agudo, a laparotomia exploradora pode estar indicada. Além disso, em algumas complicações da PA como o infarto ênteromesentérico ou a síndrome do compartimento abdominal, a intervenção cirúrgica se impõe. Em todos essas situações, operação é realizada em momentos precoces do quadro de PA, e de uma forma geral, o curso clínico desses pacientes não costuma ser muito favorável, em especial por conta de fatores como a dificuldade em se distinguir nesta fase as estruturas da região retropancreática devido ao intenso processo inflamatório local, à situação clínica geral do paciente que apresenta importante resposta inflamatória sistêmica e à infecção secundária de tecidos necróticos inicialmente estéreis.

Mais tardiamente, a intervenção cirúrgica pode ser necessária no manejo de PA grave com necrose infectada. Como já citado previamente, pacientes com este quadro devem idealmente ser submetidos à tomografia computadorizada com contraste venoso, em períodos mais tardios que sete dias, quando então o achado de necrose é mais facilmente detectado. Nessas condições, a maior parte das necroses são do tipo estéril e a probabilidade de infecção aumenta com a extensão da necrose. Na suspeita de necrose infectada, o padrãoouro para o seu diagnóstico é a punção com agulha fina guiada por tomografia computadorizada, seguida de gram e cultura do aspirado. Uma vez confirmado o diagnóstico, o tratamento com antibioticoterapia deve ser instituído, mas algum tipo de intervenção cirúrgica deve ser obrigatoriamente empregado, uma vez que a antibioticoterapia isolada nesses pacientes acarreta alta mortalidade ${ }^{10}$. Sendo assim, classicamente, o tratamento padrão desses pacientes envolve a realização de laparotomia exploradora com necrosectomia pancreática através da dissecção digital ou instrumentação delicada, desbridamento e lavagem do tecido peripancreático, com resultados já bem sedimentados em séries extensas ${ }^{40}$. Após isso, o abdome pode ser mantido em peritoneostomia ou então fechado e mantido em sistema de lavagem contínua ${ }^{39}$.

De forma mais recente, discute-se o emprego de métodos alternativos de abordagem cirúrgica e o momento da mesma. Sendo assim, uma intervenção mais tardia teria a vantagem de permitir melhor delineamento da área de necrose ou mesmo a liquefação da mesma, resultando na formação de abcesso pancreático, cuja resolução é mais fácil, podendo ser tratado inclusive através da simples drenagem percutânea. Corroborando a isso, a intervenção precoce está associada à mortalidade maior e a número maior de procedimentos desnecessários ${ }^{23}$. Por outro lado, infelizmente, grande parte dos pacientes portadores de PA grave apresentam quadro clínico com deterioração rápida e demandam intervenção cirúrgica rápida, muitas vezes não permitindo sequer que se aguarde a confirmação da presença de infecção, por exemplo.

Como inovações técnicas, propõem-se abordagens alternativas como o acesso retroperitoneal com ou sem auxilio vídeo-laparoscópico (desbridamento retroperitoneal vídeo-assistido), com a vantagem teórica de não se violar a cavidade peritoneal, e ainda o desbridamento e a drenagem por métodos endoscópicos ou através da cirurgia por orifícios naturais ${ }^{19,37,42,47}$. De forma ainda mais recente, abordagens percutâneas envolvendo a punção guiada por tomografia computadorizada, seguida de dilatação progressiva e instrumentação do trato também tem se mostrado eficazes em número limitado de pacientes 4 . Na mesma linha, Escorrou e colaboradores ${ }^{15}$, adotaram como modalidade terapêutica de primeira escolha a necrosectomia por via transgástrica. Em série recente, os autores descrevem sucesso terapêutico em 13 casos de necroses pancreáticas submetidas à necrosectomia por essa via. Segundo a metodologia dos autores, uma abertura na parede posterior do estômago era realizada permitindo o acesso do endoscópio à área de necrose. $\mathrm{A}$ partir disso, um amplo desbridamento torna-se factível, não sendo necessária a repetição do procedimento 
em nenhum dos casos relatados. Uma vantagem adicional dessa técnica seria também de se evitar a devastadora complicação da fístula pancreato-cutânea, comum em abordagens tradicionais e percutâneas. Embora promissoras, essas técnicas claramente não permitem acesso tão seguro à glândula e não permitem a realização de necrosectomias extensas ou em localizações tecnicamente mais complexas como na cabeça do pâncreas, além de envolverem vias de acesso pelas quais poucos cirurgiões estão habituados a trabalhar. Desta forma, há ainda aceitação mais limitada das mesmas, indicando-se tais técnicas mais claramente em casos menos graves e com necroses mais limitadas ou melhor delineadas ${ }^{12,24}$.

Embora tenham papel fundamental no contexto desses pacientes, os métodos de necrosectomia trazem consigo riscos consideráveis. É relatada taxa de complicações de até $62 \%$, com especial destaque para a ocorrência de fístula pancreática, estenose biliar e pseudocistos, estenose do ducto pancreático resultando em pancreatite recorrente, além de insuficiência glandular endócrina ou exócrina ${ }^{1}$. De uma forma mais geral, a PA necro-hemorrágica traz consigo risco elevado de desenvolvimento de insuficiência glandular, que tende a se recuperar parcial ou totalmente após o primeiro ano, e altas taxas de re-internação por motivos diversos, dentre os quais se destaca a dor pancreática crônica ${ }^{2,43}$.

\section{CONCLUSÕES}

A PA grave é quadro que ainda muito desafia a comunidade médica internacional. Consequentemente, um grande número de trabalhos sobre esse assunto tem sido publicado na última década. No que tange ao aspecto das perspectivas futuras, destacamse o grande número de trabalhos experimentais objetivando-se avaliar terapias que interrompam a cascata inflamatória desencadeada na fisiopatogenia da doença. Em especial, moléculas como o Infliximab (Inticorpo contra o fator de necrose tumoral, TNF) e antioxidantes como o Resveratrol têm mostrado resultados promissores nesse sentido ${ }^{17}$. De forma análoga, caracterizou-se que grande parte dos fatores etiológicos desencadeantes de PA grave têm como mecanismo de ação comum a ativação do fator de transcrição nuclear- $\mathrm{KB}$ (NF- $\mathrm{KB}$ ), envolvido em processos de resposta imune e inflamatória. $A$ ativação desse fator desencadeia inúmeras respostas como a produção de radicais livres, citocinas e a proliferação de linfócitos. Sendo assim, trabalhos recentes vêm destacando o papel de moléculas inibidoras da ativação de NF- $\kappa B$, com especial destaque para a Leflunomida, como atenuantes importantes da agressão induzida pela $\mathrm{PA}^{29}$. Finalmente, tem-se apontado também um papel de destaque da oxigenioterapia hiperbárica em reduzir a lesão por estresse oxidativo das espécies reativas de oxigênio característica da PA grave ${ }^{29}$.
Do ponto de vista cirúrgico, as maiores perspectivas relacionadas ao tratamento da PA são as relacionadas ao desenvolvimento das diferentes modalidades alternativas de tratamento da necrose infectada e das demais lesões correlatas. Comojá relatado previamente, há basicamente cinco modalidades alternativas em implementação: a drenagem percutânea, que envolve o auxílio de algum método de imagem e permite a instrumentação limitada do tecido pancreático por meio da dilatação progressiva do acesso obtido; a abordagem endoscópica "tradicional", por meio da punção transgástrica ou transduodenal, que tem a vantagem de ser pouco invasiva e mórbida, mas permite intervenção mais limitada e, portanto, ineficaz quando há necessidade de desbridamentos mais amplos e de coleções menos fluidas; a cirurgia por orifícios naturais (NOTES), que nesse caso utiliza o acesso transgástrico, evitando assim as incisões de parede abdominal e permitindo acesso mais seguro e instrumentação mais ampla do tecido necrótico do que as técnicas endoscópicas por punção ${ }^{25 ;}$; desbridamento laparoscópico, que possui alta eficácia em localizações específicas das coleções, como na goteira parieto-cólica e bolsa omental, mas que só pode ser aplicado em situações nas quais o paciente tolere o pneumoperitônio necessário para o emprego da técnica; e, por fim, a abordagem retroperitoneal, que permite acesso amplo a áreas não contempladas pelas demais técnicas e o seu desbridamento completo, mas com a desvantagem de ser mais invasiva que elas $^{35}$. O emprego dessas novas metodologias tem sido realizado de forma cada vez mais liberal e muitos centros elegem alguma delas como de primeira escolha para a abordagem das necroses infectadas, deixando a abordagem cirúrgica tradicional apenas para os casos em que há falha com estes procedimentos ${ }^{4}$.

\section{REFERÊNCIAS}

1. Amano H, Takada T, Isaji S, Takeyama $Y$, Hirata K, Yoshida M, Mayumi T, Yamanouchi E, Gabata T, Kadoya M, Hattori T, Hirota M, Kimura Y, Takeda K, Wada K, Sekimoto M, Kiriyama S, Yokoe M, Hirota M, Arata S. Therapeutic intervention and surgery of acute pancreatitis.J Hepatobiliary Pancreat Sci. 2010 Jan;17(1):53-9.

2. Ardengh JC, Coelho DE, Santos JS, Módena JLP, Eulalio JMR, Coelho JF. Pancreatite aguda sem etiologia aparente: a microlitíase deve ser pesquisada? Rev Col Bras Cir. 2009;36(5):449-58.

3. Ayub K, Slavin J, Imada R. Endoscopic retrograde cholangiopancreatography in gallstone-associated acute pancreatitis. Cochrane Database Syst Rev. 2010 Jan 20;(1):CD003630.

4. Bala M, Almogy G, Klimov A, Rivkind AI, Verstandig A. Percutaneous "stepped" drainage technique for infected pancreatic necrosis. Surg Laparosc Endosc Percutan Tech. 2009 Aug;19(4):e113-8.

5. Banks PA, Gerzof SG, Langevin RE, Silverman SG, Sica GT, Hughes MD. CT-guided aspiration of suspected pancreatic infection: bacteriology and clinical outcome. Int J Pancreatol. 1995 Dec;18(3):265-70.

6. Beger HG, Bittner R, Block S, Büchler M. Bacterial contamination of pancreatic necrosis. A prospective clinical study. Gastroenterology. 1986 Aug;91(2):433-8.

7. Bradley EL 3rd, Dexter ND. Management of severe acute pancreatitis: a surgical odyssey. Ann Surg. 2010 Jan;251(1):6-17. 
8. Bradley EL 3rd. A clinically based classification system for acute pancreatitis. Summary of the International Symposium on Acute Pancreatitis, Atlanta, Ga, September 11 through 13, 1992. Arch Surg. 1993 May; 128(5):586-90.

9. Büchler MW, Gloor B, Müller CA, Friess H, Seiler CA, Uhl W. Acute necrotizing pancreatitis: treatment strategy according to the status of infection. Ann Surg. 2000 Nov;232(5):619-26.

10. Charbonney E, Nathens AB. Severe acute pancreatitis: A review. Surg Infections 2008; 9(6):573-8.

11. Chooklin S, Pereyaslov A, Bihalskyy I. Pathogenic role of myeloperoxidase in acute pancreatitis. Hepatobiliary Pancreat Dis Int 2009; 8:627-31.

12. Connor $\mathrm{S}$, Ghaneh $\mathrm{P}$, Raraty $\mathrm{M}$, Sutton R, Rosso E, Garvey CJ, Hughes ML, Evans JC, Rowlands P, Neoptolemos JP. Minimally invasive retroperitoneal pancreatic necrosectomy. Dig Surg. 2003;20(4):270-7.

13. Dambrauskas Z, Gulbinas A, Pundzius J, Barauskas G. Value of routine clinical tests in predicting the development of infected pancreatic necrosis in severe acute pancreatitis. Scand J Gastroenterol. 2007 Oct;42(10):1256-64.

14. Dellinger EP, Tellado JM, Soto NE, Ashley SW, Barie PS, Dugernier $\mathrm{T}$, Imrie CW, Johnson CD, Knaebel HP, Laterre PF, Maravi-Poma $\mathrm{E}_{\text {, }}$ Kissler JJ, Sanchez-Garcia M, Utzolino S. Early antibiotic treatment for severe acute necrotizing pancreatitis: a randomized, doubleblind, placebo-controlled study. Ann Surg. 2007 May;245(5):674-83.

15. Escourrou J, Shehab H, Buscail L, Bournet B, Andrau P, Moreau J, Fourtanier G. Peroral transgastric/transduodenal necrosectomy: success in the treatment of infected pancreatic necrosis. Ann Surg. 2008 Dec;248(6):1074-80.

16. Fan ST, Lai EC, Mok FP, Lo CM, Zheng SS, Wong J. Early treatment of acute biliary pancreatitis by endoscopic papillotomy. N Engl J Med. 1993 Jan 28;328(4):228-32.

17. Fantini L, Tomassetti P, Pezzilli R. Management of acute pancreatitis: current knowledge and future perspectives. World J Emerg Surg. 2006 May 23;1:16.

18. Fölsch UR, Nitsche R, Lüdtke R, Hilgers RA, Creutzfeldt W. Early ERCP and papillotomy compared with conservative treatment for acute biliary pancreatitis. The German Study Group on Acute Biliary Pancreatitis. N Engl J Med. 1997 Jan 23;336(4):237-42.

19. Friedland S, Kaltenbach T, Sugimoto M, Soektikno R. Endoscopic necrosectomy of organized pancreatic necrosis: a currently practiced NOTES procedure. J Hepatobiliary Pancreat Surg 2009; 16:266-9.

20. Frossard JL, Steer M, Pastor CM. Acute pancreatitis. Lancet 2008; 371:143-52.

21. Gloor B, Müller CA, Worni M, Stahel PF, Redaelli C, Uhl W, Büchler MW. Pancreatic infection in severe pancreatitis: the role of fungus and multiresistant organisms. Arch Surg. 2001 May;136(5):592-6.

22. Gupta R, Wig JD, Bhasin DK, Singh P, Suri S, Kang M, Rana SS, Rana S. Severe acute pancreatitis: The life after. J Gastrointest Surg 2009; 13:1328-36.

23. Hartwig W, Maksan SM, Foitzik T, Schmidt J, Herfarth C, Klar E. Reduction in mortality with delayed surgical therapy of severe pancreatitis. J Gastrointest Surg. 2002;6(3):481-7.

24. Horvath KD, Kao LS, Wherry KL, Pellegrini CA, Sinanan MN. A technique for laparoscopic-assisted percutaneous drainage of infected pancreatic necrosis and pancreatic abscess. Surg Endosc. 2001 Oct;15(10):1221-5

25. Isayama $H$, Yamamoto $K$, Mizuno $S$, Yashima $Y$, Togawa O, Kogure H, Sasaki T, Sasahira N, Nakai Y, Hirano K, Tsujino T, Tada M, Kawabe T, Omata M. NOTES and endoscopic pancreatic necrosectomy for the GI endoscopist. J Hepatobiliary Pancreat Surg 2009; 16:270-3.

26. Jafri NS, Mahid SS, Idstein SR, Hornung CA, Galandiuk S. Antibiotic prophylaxis is not protective in severe acute pancreatitis: a systematic review and meta-analysis. Am J Surgery 2009; 197:806-13.

27. Karimgani I, Porter KA, Langevin RE, Banks PA. Prognostic factors in sterile pancreatic necrosis. Gastroenterology. 1992 Nov;103(5):1636-40.

28. Kochhar R, Ahammed SK, Chakrabarti A, Ray P, Sinha SK, Dutta U, Wig JD, Singh K. Prevalence and outcome of fungal infection in patients with severe acute pancreatitis. J Gastroenterol Hepatol. 2009 May;24(5):743-7
29. Kutluana U, Oruc N, Nart D, Kaptanoglu B, Yonetci N, Ozutemiz O. Leflunomide. Is a new oral agent in treatment of acute pancreatitis? Pancreas 2010; 39(2):237-42.

30. Luiten EJ, Hop WC, Lange JF, Bruining HA. Controlled clinical trial of selective decontamination for the treatment of severe acute pancreatitis. Ann Surg. 1995 Jul;222(1):57-65.

31. Marik PE, Zaloga GP. Meta-analysis of parenteral nutrition versus enteral nutrition in patients with acute pancreatitis. BMJ 2004; 328:1407-10.

32. Mazaki T, Ishii $\mathrm{Y}$, Takayama T. Meta-analysis of prophylactic antibiotic use in acute necrotizing pancreatitis. Br J Surg 2006; 93:674-84

33. Targarona Modena J, Barreda Cevasco L, Arroyo Basto C, Orellana Vicuña A, Portanova Ramírez M. Total enteral nutrition as prophylactic therapy for pancreatic necrosis infection in severe acute pancreatitis. Pancreatology. 2006;6(1-2):58-64.

34. Mofidi R, Suttie SA, Patil PV, Ogston S, Parks RW. The value of procalcitonin at predicting the severity of acute pancreatitis and development of infected pancreatic necrosis: Systematic review. Surgery 2009; 146(1):72:81.

35. Navaneethan E, Vege SS, Chari ST, Baron TH. Minimally invasive techniques in pancreatic necrosis. Pancreas 2009; 38(8): 867-75.

36. Neoptolemos JP, Carr-Locke DL, London NJ, Bailey IA, James D, Fossard DP. Controlled trial of urgent endoscopic retrograde cholangiopancreatography and endoscopic sphincterotomy versus conservative treatment for acute pancreatitis due to gallstones. Lancet. 1988 Oct 29;2(8618):979-83.

37. Papachristou GI, Takahashi N, Chahal P, Sarr MG, Baron TH. Peroral endoscopic drainage/debridement of walled-off pancreatic necrosis. Ann Surg 2007; 245:943-51.

38. Paye F, Rotman N, Radier C, Nouira R, Fagniez PL. Percutaneous aspiration for bacteriological studies in patients with necrotizing pancreatitis. Br J Surg. 1998 Jun;85(6):755-9.

39. Rau B, Bothe A, Beger HG. Surgical treatment of necrotizing pancreatitis and closed lavage: changing patient characteristics and outcome in a 19-year, single-center series. Surgery 2005; 138:28-39.

40. Rodriguez JR, Razo AO, Targarona J, Thayer SP, Rattner DW, Warshaw AL, Fernández-del Castillo C. Debridement and closed packing for sterile or infected necrotizing pancreatitis: insights into indications and outcomes in 167 patients. Ann Surg. 2008 Feb;247(2):294-9.

41. Sawa H, Ueda T, Takeyama Y, Yasuda T, Shinzeki M, Matsumura N, Nakajima T, Matsumoto I, Fujita T, Ajiki T, Fujino Y, Kuroda Y. Treatment outcome of selective digestive decontamination and enteral nutrition in patients with severe acute pancreatitis. J Hepatobiliary Pancreat Surg. 2007;14(5):503-8.

42. Seifert $H$, Wehrmann T, Schmitt $T$, Zeuzem S, Caspary WF. Retroperitoneal endoscopic debridement for infected peripancreatic necrosis. Lancet. 2000 Aug 19;356(9230):653-5.

43. Speranzini MB, Bassi DG, Cauduro AB, Pezzolo S. Estenose ductal pancreática por trauma: tratamento pela anastomose pancreatojejunal. ABCD Arq Bras Cir Dig. 2004;17(1):67-70.

44. Tsui N-C, Zhao E, Li ZL, Miao B, Cui YF, Shen YF, Qu PF. Microbiological findings in secundary infection of severe acute pancreatitis. A retrospective clinical study. Pancreas 2009; 38:499-502.

45. Uhl W, Warshaw A, Imrie C, Bassi C, McKay CJ, Lankisch PG, Carter R, Di Magno E, Banks PA, Whitcomb DC, Dervenis $C$, Ulrich $C D$, Satake K, Ghaneh P, Hartwig W, Werner J, McEntee G, Neoptolemos JP, Büchler MW. IAP Guidelines for the Surgical Management of Acute Pancreatitis. Pancreatology. 2002;2(6):565-73.

46. Van Vyve EL, Reynaert MS, Lengele BG, Pringot JT, Otte JB, Kestens PJ. Retroperitoneal laparostomy: a surgical treatment of pancreatic abscesses after an acute necrotizing pancreatitis. Surgery. 1992 Apr;111(4):369-75.

47. Winslet MC, Imray C, Neoptolemos JP. Biliary acute pancreatitis. Hepatogastroenterology 1991; 38:120-123. 